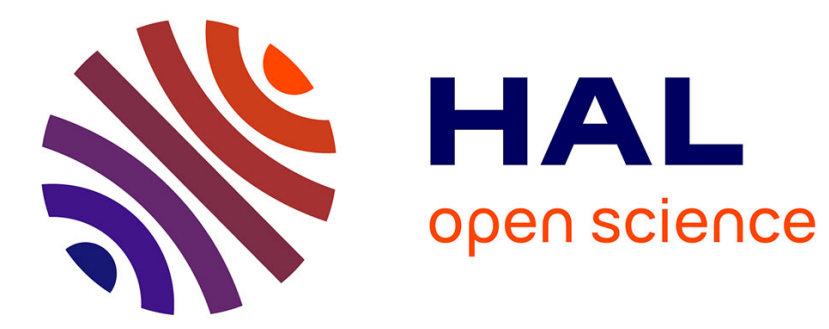

\title{
Walking with virtual people: Evaluation of locomotion interfaces in dynamic environments
}

\author{
Anne-Hélène Olivier, Julien Bruneau, Richard Kulpa, Julien Pettré
}

\section{To cite this version:}

Anne-Hélène Olivier, Julien Bruneau, Richard Kulpa, Julien Pettré. Walking with virtual people: Evaluation of locomotion interfaces in dynamic environments. IEEE Transactions on Visualization and Computer Graphics, 2018, 24 (7), pp.2251-2263. 10.1109/TVCG.2017.2714665 . hal-01557761

\section{HAL Id: hal-01557761 \\ https://hal.inria.fr/hal-01557761}

Submitted on 6 Jul 2017

HAL is a multi-disciplinary open access archive for the deposit and dissemination of scientific research documents, whether they are published or not. The documents may come from teaching and research institutions in France or abroad, or from public or private research centers.
L'archive ouverte pluridisciplinaire HAL, est destinée au dépôt et à la diffusion de documents scientifiques de niveau recherche, publiés ou non, émanant des établissements d'enseignement et de recherche français ou étrangers, des laboratoires publics ou privés. 


\title{
Walking with virtual people: Evaluation of locomotion interfaces in dynamic environments
}

\author{
Anne-Hélène Olivier, Julien Bruneau, Richard Kulpa, and Julien Pettré Member, IEEE
}

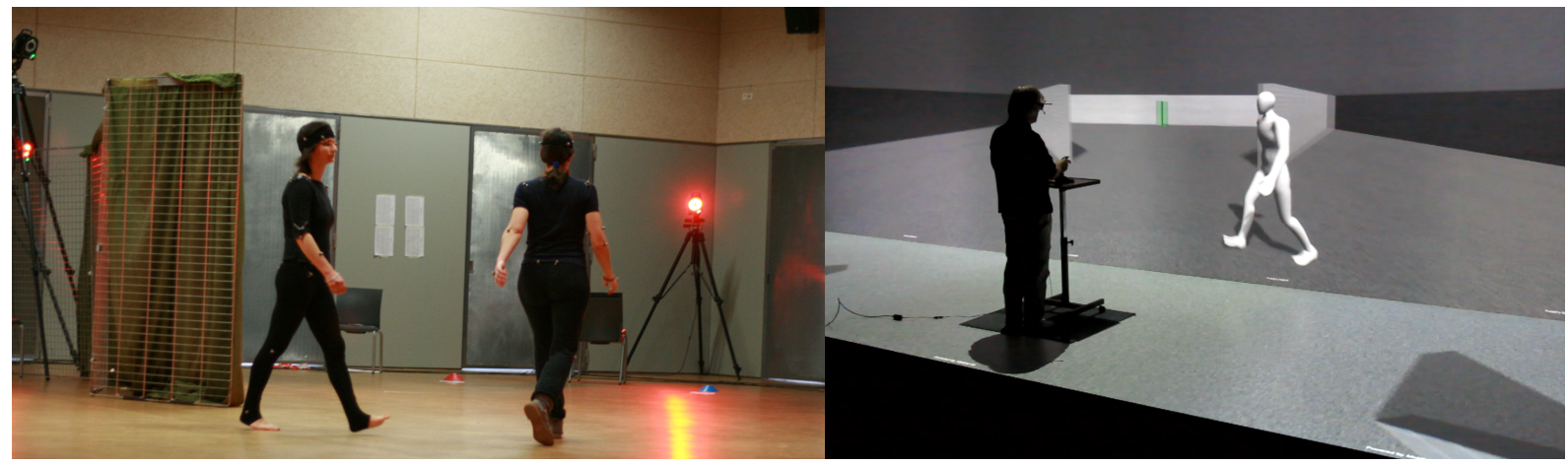

Fig. 1. The objective of this article is to evaluate whether small scale interactions during locomotion between a real user and a virtual human (right) are similar in virtual conditions in comparison with real ones (left).

\begin{abstract}
Navigating in virtual environments requires using some locomotion interfaces, especially when the dimensions of the environments exceed the ones of the Virtual Reality system. Locomotion interfaces induce some biases both in the perception of the self-motion or in the formation of virtual locomotion trajectories. These biases have been mostly evaluated in the context of static environments, and studies need to be revisited in the new context of populated environments where users interact with virtual characters. We focus on situations of collision avoidance between a real participant and a virtual character, and compared it to previous studies on real walkers. Our results show that, as in reality, the risk of future collision is accurately anticipated by participants, however with delay. We also show that collision avoidance trajectories formed in VR have common properties with real ones, with some quantitative differences in avoidance distances. More generally, our evaluation demonstrates that reliable results can be obtained for qualitative analysis of small scale interactions in VR. We discuss these results in the perspective of a VR platform for large scale interaction applications, such as in a crowd, for which real data are difficult to gather.
\end{abstract}

Index Terms-Locomotion, Interaction, Evaluation, Experiment, Collision Avoidance, Virtual Reality.

\section{INTRODUCTION}

Navigation is a fundamental requirement in many Virtual Reality (VR) applications. A lot of research has been devoted to the design of efficient locomotion techniques to virtually walk in immersive 3D environments. Especially, the level of realism of the locomotion trajectories formed by some locomotion interfaces as well as the accuracy of the perception of the virtual travel was evaluated. Nevertheless, these questions of realism and perception were generally addressed in static 3D environments, empty of any virtual people. Among many possible applications, we aim at using $3 \mathrm{D}$ environments populated with virtual characters to study individual locomotion behaviors in crowds. Then, the question of realism and perception using various locomotion techniques needs revisiting in the new context of interactions with virtual characters.

The main purpose of this article is to investigate whether

- Anne-Hélène Olivier, Richard Kulpa, Julien Bruneau and Julien Pettré are with INRIA Rennes, France. E-mail: first.last@inria.fr.

- Anne-Hélène Olivier and Richard Kulpa are with M2S lab, University Rennes 2, France. E-mail: first.last@univ-rennes2.fr.

Manuscript received 31 Mar. 2015; accepted 1 Aug. 2015; date of publication xx Aug. 2015; date of current version 25 Oct. 2015. For information on obtaining reprints of this article, please send e-mail to:tvcg@computer.org. small scale interactions between a real user and a virtual human during locomotion tasks are preserved in VR compared to interactions in real conditions. The long term objective is to extend these results to larger scale interactions, such as in a crowd, for which real human data are difficult to gather. Our objective is a wide one. In this article we focus our study on the situation of collision avoidance between a real human and a virtual character.

A lot of research has been performed in Biomechanics to describe the collision avoidance interaction, which can be used as a reference for evaluation purposes. More specifically, it has been shown that humans accurately perceive others' motion and risk of collision, allowing them to perform anticipated adaptations of their trajectories. As a result, realistic interactions between a real human and a virtual character first require that the relative motion with the virtual character is correctly perceived, and risk of future collision detected. Second, it is required that the employed locomotion interface does not induce biases in the avoidance trajectories.

The contributions of this article are as follows:

1. we experimentally evaluate the ability of a real user to accurately perceive the risk of collision with a moving character. We show that the level of perception is accurate enough to expect realistic interactions 
2. we experimentally compare various basic locomotion techniques and show how they influence the virtually formed locomotion trajectories.

3. we provide recommendations for the design of VR platforms to perform human locomotion studies in the context of interactions.

The remaining of the article is organized as follows. Section 2 describes related work with respect to the use of VR to study human behavior as well as human interactions during locomotion. Section 3 is the overview of the article and presents the objectives, the approach we proposed as well as the general description of the experiments we conducted. Sections 4 and 5 describe respectively the experiments evaluating the perception of the risk of collision in VR and the user's behavior to avoid a collision with a virtual human. Finally, the results are discussed in Section 6.

\section{RELATED WORK}

\subsection{VR-based human behavior studies}

VR-based experimental platforms are powerful tools to perform perception-action experiments [24]. They have been used more and more to analyze sport motions [4], motion control [14], perceptual control laws [48], or spatial cognition [26, 27]. VR is used in such experiments because it allows experimenters to expose a population to fully controlled stimuli, conditions can be accurately repeated from trial to trial, stimuli can be interactive and adapted to participants' responses, factors can generally be isolated and studied independently, virtual reality can be manipulated to this end, etc. In this context, our long term objective is to develop a VR experimental platform to study individual locomotion behaviors in crowds with the aim of developing new crowd simulation algorithms.

Studying human motion in crowds requires large virtual environments, larger than any CAVE. This prevents users from freely walking in the virtual environment and constraints the use of locomotion interfaces. A locomotion interface is made up of two main components, a locomotion metaphor (device) and a transfer function (software). Both components may introduce bias in the generated virtual motion. Several devices can be used as locomotion interfaces [17] such as joysticks, treadmills [40], the Joyman [34] or the Virtualizer (http: // cyberith.com/product/). None of these devices stimulate all of the sensory channels involved in locomotion in a realistic manner. Also, specific techniques were developed to maximize the virtually reachable space, such as "walking-inplace" $[39,44]$ or "redirected walking" paradigms [36, 41, 29] which trick participants to walk in circles in the real environment while moving straight in the virtual one or the "computermediated optic flow" which can modify self motion perception [6]. Locomotion interfaces are not the only sources of bias in experiments. VR platforms are designed to visually reproduce real environments, but displays affect the available information. For example, there are differences in perceived velocity [2], walking speed is decreased [14], personal space size is modified [16] and walking in VR is performed with increased instability [18].
All these studies stress the need for validating VR tools before using VR for capturing and analyzing human motion data. Several studies evaluated the influence of VR on human perception and behavior. From a perceptual and cognitive point of view, authors reported for example a distance compression in VR $[23,50,37]$ but this effect is reduced after five minutes of continuous visual feedback [28]. Authors also used presence and cybersickness questionnaires [39, 51, 49, 43] in order to gather more subjective data from the users. From a locomotor behavioral point of view, researchers proposed several metrics to evaluate the trajectories generated in VR. An interesting and objective approach for such an evaluation is to compare trajectories performed under various VR conditions (locomotion interfaces, control laws, viewing conditions...) with trajectories performed in the real world. Studies on novel locomotion interfaces evaluate virtual trajectories using performance criteria [40, 51] (i.e., task completion time, traveled distance, walking speed, steps characteristics...) or empirical observations of the trajectory [51]. Ruddle et al. [38] also showed that speed profile is an important clue to evaluate user proficiency in the task. Fink et al. [14] used a different set of metrics, namely the mean radius of curvature along the full path, the maximum euclidean distance from a straight line between the origin and the target, and the minimum euclidean distance between the path and the obstacles of the virtual environment. Whitton et al.[49] used Principal Component Analysis to study a set of VR trajectories, and found that for their specific constrained task velocity profiles were mostly defined by the maximum velocity, the percent of time to reach the maximum velocity, and the maximum deceleration. Cirio et al. [10] evaluated the realism of unconstrained goal directed trajectories performed in VR using both geometrical and temporal evaluation criteria (collisions, duration, tangential and angular velocity profile, shape...). Using these comparison metrics, we are able to chose the conditions of the VR platform that maximize the realism of the trajectory performed [10]. Fink et al. [14] concluded that despite the differences between virtual and real environment, the data gathered with a VR platform can still be useful when carefully considered.

There were a lot of studies concerning locomotion in VR [42] as well as concerning the evaluation of walking trajectories in VR. However, few of them took interest in the locomotion trajectories performed during interactions with a virtual human. Some studies have explored the influence of the nature of a static obstacle (human/box, real/virtual) in the interacting locomotion trajectory [1] or in an other task have investigated the avoidance of moving obstacle in a bicycling task in VR [8]. Our study focuses on the collision avoidance task between a real user and a virtual human. Our approach follows similar principles to previous evaluations of locomotion interfaces $[10,38]$. Major differences are that we consider the perception of some virtual character's motion, and the bias induced by locomotion interfaces on adaptations (and not formation) of locomotion trajectories.

\subsection{Human interactions during locomotion}

Human interactions during locomotion have been analyzed considering several kinds of situations such as following be- 
haviour [22], side-by-side [33] or face-to-face walking [12] with another walker, group formation [20], or collision avoidance. The latter situation received a lot of attention. Authors studied the nature of information taken by a walker to predict if there will be a collision and when it will occur [11], the adaptations a walker performs to step over [32] or circumvent [45] a static obstacle, or described the interactions between walkers and their environment as coupled dynamical systems [47, 13] where heading is adapted according to the distance and the angle to stationary goals and obstacles.

Other works focused on the avoidance of passive moving obstacles. To avoid a moving mannequin on a $45^{\circ}$ collision path, human walkers adapt their motion both in the antero-posterior and medio-lateral planes [15] to preserve their personal space, defined as an elliptic subjective area around the walker. When a participant and a mannequin are walking face-to-face, a 2-step avoidance strategy is observed: first, a change in heading and second, an adjustment of walking speed [9] with adaptations always initiated at a constant distance from the obstacle.

When the passive obstacle is a confederate human on a $90^{\circ}$ collision path in a quite small area, a walker favors speed changes even if it is not an optimal solution [3]. Using a larger area, authors demonstrated that the walker's adaptations depend on crossing angle (from acute to obtus angles) and walking speed conditions (slow, comfortable and fast speeds) [19]: path is always adapted; speed is adapted only for acute angles and its adaptations depend on the walking speed conditions.

Finally, few authors took interest in the more complex case of collision avoidance between two walkers. For face-to-face interactions, authors showed that gender and height influence the collision avoidance strategies [46]. For $90^{\circ}$ crossings, walkers accurately estimate the future risk of collision since they react only when required and they solve the collision avoidance task with anticipation [31]. Moreover, these adaptations are performed collaboratively but they are role-dependent [30]. Indeed, the crossing order impacts both the contribution and the strategies used: the participant giving way contributes more than the one passing first to avoid the collision. Both walkers reorient their path but the participant giving way also adapts his speed. These latter two studies [30, 31] proposed a detailed metric to quantify interaction between two walkers in a collision avoidance task.

In this article, our intention is not to extend knowledge on human interactions during locomotion. However, we want to benefit from the last studies on collision avoidance between two walkers. We aim at studying similar situations in the context of interaction between a real human and a virtual character. We further describe the method and result presented in $[30,31]$ in Section 3.2. We propose to use these results as a baseline to evaluate the interactions performed in VR conditions.

\section{OVERVIEW}

\subsection{Objectives}

Figure 2 displays a VR interaction loop between a real user and a virtual character and its main technical components. The

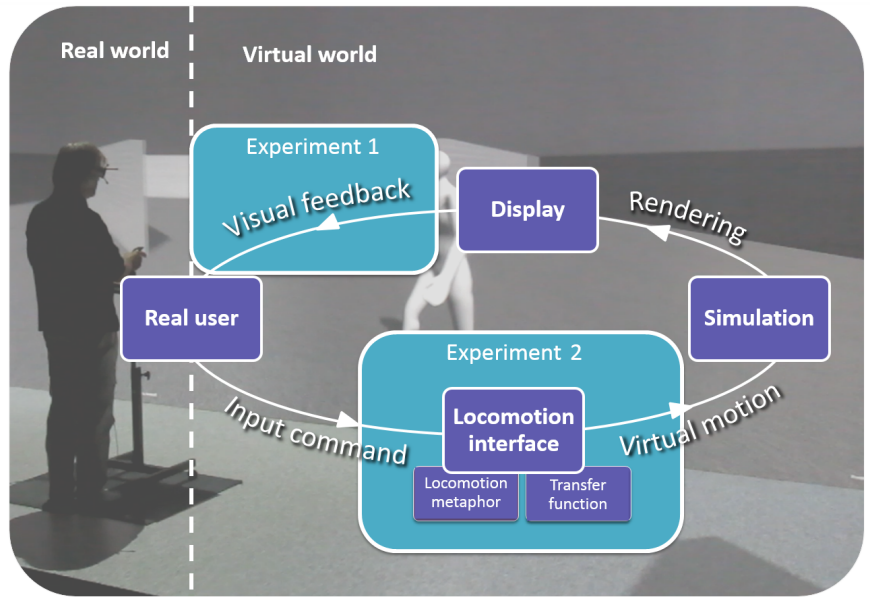

Fig. 2. Illustration of the interaction loop between a real user and a virtual character in an immersive environment.

purpose of this study is to investigate whether small scale interactions between a real user and a virtual human during a locomotor task are preserved in VR. Our question is "Do users avoid collision with the virtual character by performing the same adaptations to their motion than what they would have done in real conditions?".

\subsection{Approach}

Real interactions between two walkers were studied in [30, 31]. Results show that humans adapt their trajectories when, and only when, a risk of future collision is detected. This future risk of collision is estimated by computing the future distance of closest approach, assuming that walkers keep constant velocity (speed and direction). Adaptations are performed to cancel risk of collision and pass at respectful distance from others. What about interactions between real and virtual walkers? We evaluate the VR interaction loop (Figure 2) by comparing interactions performed by a real participant interacting with a virtual walker with ones performed by 2 real walkers. We propose a 2-step experiments. Experiment 1 (Section 4) is a perception study: the participant moves in the VR environment where a virtual character is also moving. The participant is asked about risk of collision with the virtual character. This step ensures that the visual feedback provided to users enable them to correctly perceive risk of future collision as in real conditions. Experiment 2 (Section 5) considers various locomotion interfaces and their influence on the metrics of interaction.

To enable comparisons, we consider a situation of interaction (relative motion and position) close to the one studied in [30,31], which focused on collision avoidance task during $90^{\circ}$ crossings between two walkers in a large area $(15 \mathrm{~m} \times 15 \mathrm{~m})$. They set occluding walls ( $2 \mathrm{~m}$ high by $3 \mathrm{~m}$ long) in the area so that walkers cannot see each other before reaching their natural speeds. Our comparison criteria are based on the mpd function analysis ( $m p d$ means minimum predicted distance) introduced in [31]. $m p d$ is a continuous function of time which computes the future distance of closest approach between two walkers based on their current position, orientation and speed. Extrapolation of future positions assumes that walkers keep walking at the same orientation and speed. mpd function is ana- 
lyzed over the interaction period of time, which ranges from the time walkers are able to see each other to the time they reach the closest distance. $m p d$ is constant in time when walkers do not perform adaptations of their respective trajectories (i.e., changes in speed or orientation resulting in variations of $m p d)$. mpd thus reveals the effect of adaptations on the future crossing distance as well as the temporal structure of collision avoidance. It was shown that the reaction period takes place when $m p d$ increases due to walkers' adaptations [31]. It is preceded by an observation period ( $\mathrm{mpd}$ is constant but low), and followed by a regulation period ( $\mathrm{mpd}$ is constant but high enough for collision avoidance).

In our article, we use a signed version of the $m p d$, where the sign is determined according to the order of passage at the crossing point: negative $m p d$ means that the user gives way, whereas positive values means that the user passes first.

We formulate 2 hypotheses based on the expectation that collision avoidance behaviors in VR match the properties of real ones:

- Hypothesis \#1: user accurately estimates the collision risk with the virtual character. In real interactions, adaptations ( $m p d$ variations) are observed if, and only if, a risk of future collision exists ( $m p d$ value is low at the beginning of interactions, $<1 \mathrm{~m}$ ). We check the perception of collision risk in Experiment 1, we also analyze the evolutions of $m p d$ in Experiment 2.

- Hypothesis \#2: users anticipate collision. In real interactions, having a regulation period ( $\mathrm{mpd}$ constant with high enough value) before walkers reach the closest distance demonstrates that avoidance maneuvers are performed with anticipation. We expect to observe the same temporal structure of $m p d$ evolutions: over the average duration of interactions (normalized time), the observation period is between $0 \%$ and $10 \%$, reaction period is from $10 \%$ to $80 \%$ and the regulation period follows from $80 \%$ to $100 \%$.

\subsection{Experiments overview}

Our experiment is decomposed in two steps, called Experiment 1 and Experiment 2. They shared common elements described below.

Apparatus Experiments took place in the 4-screen CAVE illustrated in Figure 1, equipped with 13 projectors, 15MPixels resolution in total, $9 m$ wide, $3 m$ high and $3 m$ deep. 3D environment display and character animation is designed in the Unity game engine. Multi-surface rendering is performed by the MiddleVR plugin. Active stereoscopy is achieved with Volfony ActiveEyes Pro Radiofrequency wearable glasses. Glasses are tracked by an ART tracking system. The virtual environment has the same properties (i.e., size, occluding walls) than the one used in Olivier et al. studies [30, 31].

Task Participants were immersed in an environment where a character is walking. They performed a collision avoidance task in the same conditions as presented in $[30,31]$ and illustrated in Figure 3. Participants avoided a virtual character walking along a trajectory perpendicular to their own trajectory. We set

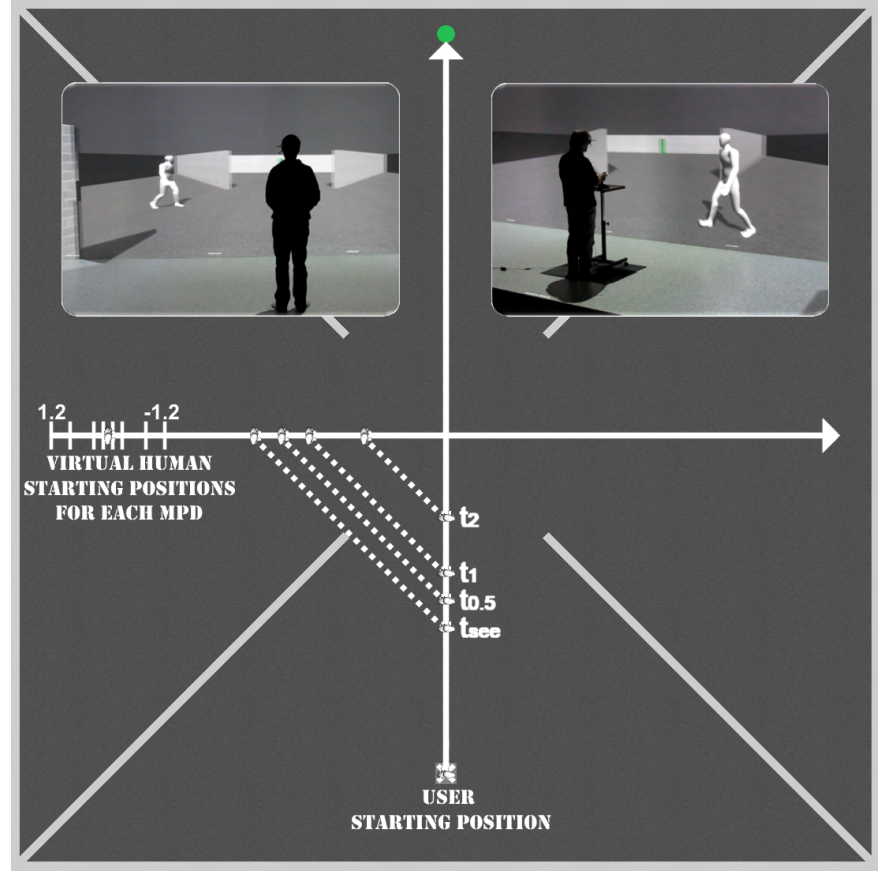

Fig. 3. Virtual environment and experimental situation used in this study. The virtual human has several starting positions corresponding to different value of $m p d$ giving the virtual human some advance or delay over the user. Position of both the user and the virtual human are represented for different times: when they can see each other $t_{\text {see }}$ and then $t_{0.5}, t_{1}$ and $t_{2}$ which are respectively $0.5 \mathrm{~s}, 1 \mathrm{~s}$ and $2 \mathrm{~s}$ after $t_{\text {see }}$ (if there is no adaptation from the user). A dotted line joins the positions of the character and of the participant at those times for $m p d=0$.

wall in the environment to control the moment at which participants can perceive the virtual character. Before they see it, the motion is initiated and participants reach a comfortable regular speed. In Experiment 1, participants are passively moving in the virtual environment. In Experiment 2 participants are asked to reach a green target by using various locomotion interfaces. Collision avoidance with the white character is part of this task. This virtual character does not react to the participants. The character has no expression (no face, no texture) and is animated based on a neutral motion captured locomotion cycle. The comfortable regular speed for the virtual character as well as for the initial motion of the participant is set to $1.4 \mathrm{~m} . \mathrm{s}^{-1}$ according to the study of Bohannon [5] on normative walking speed data. For each experiment, questions about the task were asked to participants using slides with images and text.

Conditions For both experiments, we controlled the initial value of the mpd function by shifting the character's starting position (Figure 3 ). We studied the following $m p d$ initial values: $-1.2 m,-0.8 m,-0.3 m,-0.1 m, 0.1 m, 0.3 m, 0.8 m$ and $1.2 \mathrm{~m}$. In Figure 3, the virtual human starting position is shifted to the left with a positive $m p d$ and to the right with a negative $m p d$ inducing respectively a delay or an advance over the initial user trajectory. For $m p d=\{-1.2,-0.8,0.8,1.2\} m$ there is no risk of collision (the virtual character passes first for negative values). When $m p d=\{-0.3,-0.1,0.1,0.3\} m$, initial trajectories lead to a collision and participant reaction is required. We did not study values around $0.5 m$, for which there is contact with little penetration: there is an ambiguity between contact and collision for this distance and we wanted to avoid misin- 
terpretations by participants. A trial corresponds to one crossing. There were 4 repetitions for each experimental condition ( $m p d$ conditions combined with the specific ones of each experiment). We had as many trials with the character coming from the left as from the right, but the situation is considered the same. Conditions were presented in a randomized order.

Population 15 people ( 4 women, 11 men) volunteered for the 2-step experiment. They were $27.2( \pm 7.6)$ years old (range: 20 to 52). They were naive with respect to the purpose of the experiment. All had normal or corrected-to-normal vision. They gave written and informed consent and the study conformed to the declaration of Helsinki.

\section{Experiment 1: Perception of RISK OF COLlision}

\subsection{Procedure}

Experiment 1 evaluates if users accurately perceive the risk of collision with the moving character. More precisely, we evaluate the visual feedback of the VR system: "does the user have the required visual information to correctly evaluate the risk of future collision with a moving virtual character?"

We performed the perceptual experiment illustrated in Figure 3. Participants passively moved in a VR environment along a straight trajectory. A moving character is walking along an orthogonal trajectory. We can accurately control the future distance of closest approach (e.g., $m p d$ ) of the character by delaying or advancing the character on its trajectory at initialization. Doing so, we could set the character on a collision course or not with the user, and control the intensity of this risk in terms of interpenetration volume. The smaller the absolute value of $m p d$, the higher the risk of collision.

In this experiment, the participant is only able to perceive the beginning of the virtual character motion. From the time he can see the character (after they are no longer hidden from each other by the wall), we considered 3 cutoff times: $0.5 s, 1 s$ and $2 s$. After the cutoff time, visual information is stopped by displaying a black screen. Thus the participant has a limited time to assess the situation. To verify if the collision risk was correctly perceived, the participant was then asked about it: "Will you collide with the virtual character?" "Will you pass first or give way?". For each of these questions, they had to rate their level of confidence on a 7 point Likert scale (1: not at all confident to 7: very confident). They were trained to the task 6 times before recording their answers.

Note that cutoff times were chosen based on a real experiment which showed that adaptations may start $0.5 s$ after the beginning of interactions (i.e., when participants are able to see each other) [31]. A total of 192 stimuli were displayed to each participant: 3 cutoff times, $8 \mathrm{mpd}$ values, 2 sides (from left or right) and 2 repetitions. The experiment lasted around one hour.

\subsection{Analysis}

We computed the accuracy of predicting the collision and the order of passage at the crossing for each condition (see Figure 4). We also computed the chance level threshold (expected accuracy with random answers) as follows:

$$
\text { chanceLevel }=\frac{100}{\text { NbChoices }} \pm 2 \sqrt{\frac{0.25}{\text { SampleSize }+1}} \times 100
$$

For this experiment the chance level threshold was between $37.5 \%$ and $62.5 \%$. We performed analysis of variance (ANOVA) with repeated measures on the following factors: mpd and cutoff time. Greenhouse-Geisser adjustments to the degrees of freedom were applied, when appropriate, to avoid any violation of the sphericity assumption. The effect size was computed using partial eta squared $\left(\eta_{p}^{2}\right)$. When appropriate, Bonferroni post-hoc tests were used to further analyse significant effects.
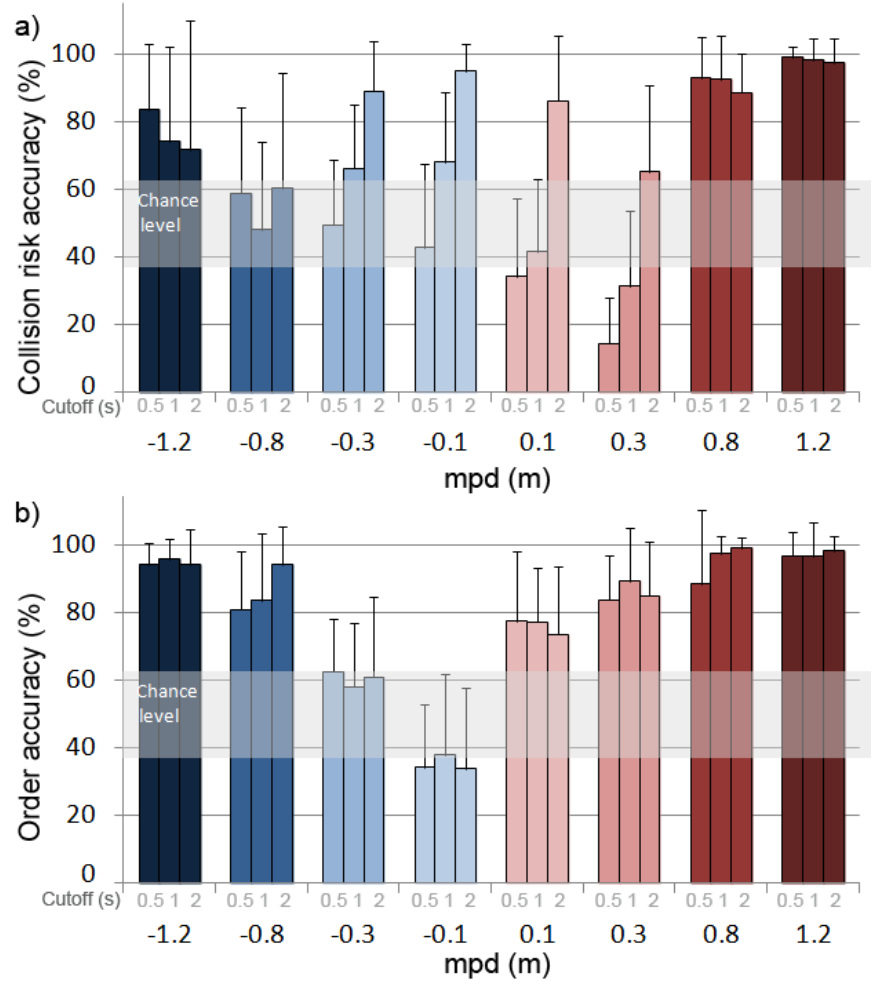

Fig. 4. Collision risk (a) and order of passage (b) judgment accuracies (mean $\pm \mathrm{SD})$.

\subsection{Results and Discussion}

Figure 4 shows the accuracy of the answers for collision (a) as well as for order of passage (b) w.r.t. mpd conditions and cutoff times. The associated levels of confidence are illustrated in Figure 5.

Risk of collision The accuracy of answers about risks of collision was influenced by the cutoff time $(F(2,28)=$ $\left.89.11, p<0.0001, \eta_{p}^{2}=0.86\right)$ and the $\operatorname{mpd}(F(2.9,41.8)=$ $\left.24.86, p<0.0001, \eta_{p}^{2}=0.64\right)$, with an interaction between these two factors $\left(F(6.1,85.9)=16.7, p<0.0001, \eta_{p}^{2}=0.54\right)$. Post hoc tests showed that the later the cutoff time, the higher the accuracy $\left(\right.$ cutoff $_{0.5 s}(59,5 \%)<$ cutoff $_{1 s}(65.1 \%)<$ cutoff $_{2 s}(81.7 \%)$ ). High accuracy was also observed for situations where $m p d$ is $0.8 \mathrm{~m}$ or $1.2 \mathrm{~m}$ and low accuracy for situations where $m p d$ is $0.3 \mathrm{~m}$. The level of confidence of participants' answer about the risk of collision was influenced by the cutoff time $(F(1.2,17.4)=37.08, p<$ $\left.0.0001, \eta_{p}^{2}=0.72\right)$ and the $m p d(F(2.6,36.6)=22.11, p<$ $0.0001, \eta_{p}^{2}=0.61$, with an interaction between these two factors $\left(F(5.3,73.9)=5.83, p<0.0001, \eta_{p}^{2}=0.29\right)$. Post hoc 

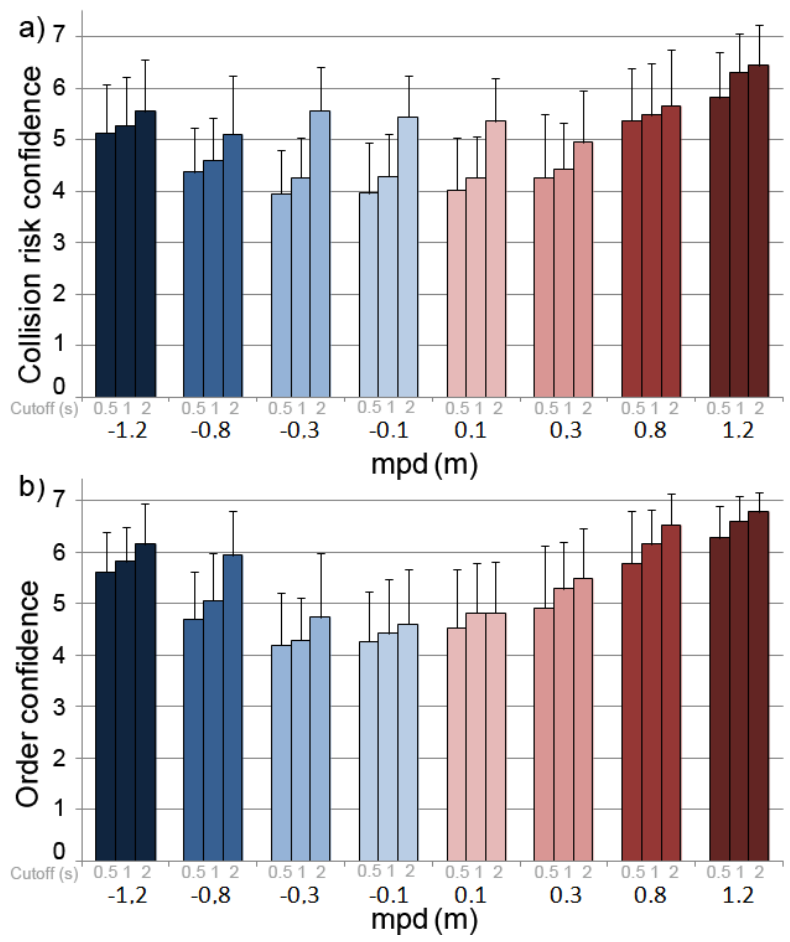

Fig. 5. Collision risk (a) and order of passage (b) judgment confidences (mean $\pm \mathrm{SD}$.

tests showed that the higher the cutoff, the higher the confidence cutoff $_{0.5 s}(4.6) \approx \operatorname{cutoff}_{1 s}(4.9)<\operatorname{cutoff}_{2 s}(5.5)$ ). Moreover, we observed higher confidence when there is actually no risk of collision (when $m p d$ is $-1.2 \mathrm{~m}, 0.8 \mathrm{~m}$ and $1.2 \mathrm{~m}$ ).

Order of passage The order of passage accuracy was only influenced by $\operatorname{mpd}\left(F(1.9,27.7)=50.35, p<0.0001, \eta_{p}^{2}=\right.$ 0.78). Post hoc tests showed that the accuracy is lower when $m p d$ is $-0.1 \mathrm{~m}$ compared to all other values. The corresponding level of confidence was influenced by the cutoff time $\left(F(1.2,16.7)=15.96, p<0.0001, \eta_{p}^{2}=0.53\right)$ and $\operatorname{mpd}\left(F(2.7,38.5)=32.81, p<0.0001, \eta_{p}^{2}=0.70\right)$. Post hoc tests showed that the higher the cutoff, the higher the confidence about order of passage (cutoff $_{0.5 s}(5)<$ cutoff $\left._{1 s}(5.3)<\operatorname{cutoff}_{2 s}(5.6)\right)$. Confidence is also higher when there is actually no risk of collision (when $m p d$ is $-1.2 \mathrm{~m},-0.8 \mathrm{~m}$, $0.8 \mathrm{~m}$ and $1.2 \mathrm{~m})$.

On average participants correctly estimated the condition of interaction with the virtual human, i.e., the risk of collision as well as the crossing order. We should however nuance this statement. The order was early identified: there was no effect of cutoff time, which means that even for a short cutoff time $(0.5 \mathrm{~s})$, participants were accurate to predict the future crossing order. The level of confidence was correlated with answers accuracy, both for order and risk of collision. Participants were conscious about their level of accuracy which is an important point. Indeed, their reaction will probably be delayed if they do not perceive the situation in a clear enough way, just as in reality.

Collision accuracy was above the chance level for all conditions only for the $2 \mathrm{~s}$ cutoff time (except for the condition $m p d=-0.8 m$ ). Actually, estimating order is equivalent to determine if the relative motion is above or below a threshold (which results into passing in front or behind). Estimating order may be simpler than estimating the risk of collision. Nevertheless, correctly estimating order is more important, as order determines which kind of adaptation improves the situation. For example to lower the risk of collision using a speed adaptation, a walker needs to decelerate when giving way and accelerate when passing first. Finally, collision accuracy was low (i.e., below the chance level) for $m p d=0.1 m$ with 0.5 s cuttoff time and for $0.3 m$ with $0.5 \mathrm{~s}$ and $1 \mathrm{~s}$ cuttoff times, collision accuracy was within the chance level for $-0.8 \mathrm{~m}$, and order accuracy was low for $m p d=-0.1 \mathrm{~m}$.

We interpret these observations as a shift in space made by the participant due to a gap in the perception of their envelope in the virtual world, as if they feel like they are ahead of their actual virtual position. Indeed, when the character is passing first with no risk of collision but close to participants $(m p d=-0.8 m)$, many participants' answers estimate that a collision will occur. At the opposite when the character passes behind the participants with a collision with small interpenetration, many participants' answers estimate that no collision will occur $(m p d=0.3 m)$. This interpretation is corroborated by feedback in questionnaires, several participants indicated that "I hardly situated myself in the virtual environment", or that "I hardly estimated the occupied volume" which "made the question about collision harder." As a conclusion, Hypothesis \#1 is validated since on average participants correctly perceive the situation of interaction with the virtual character but there were two limitations: information about collision is maybe delayed in comparison with reality, and the position in the virtual environment is perceived with an offset.

\section{EXPERIMENT 2: Collision AVOIDANCE WITH A VIR- TUAL HUMAN}

\subsection{Methods}

\subsubsection{Locomotion interfaces}

In Experiment 2, we consider the same situation of interaction with a virtual character, but let participants react to the situation: they can adapt their own motion to avoid collision with the character when they deem it necessary using a locomotion interface. We evaluate if the performed adaptations are similar to real ones. The objective is to evaluate the capacity of a given locomotion interface to induce natural behaviors.

Our objective is not to perform an exhaustive comparison of existing interfaces. Nevertheless, we evaluated 6 locomotion interfaces that use either a joystick or whole-body locomotion metaphors. First, we chose to evaluate joystick interfaces because they are commonly used and they were proved to generate realistic virtual locomotion trajectories in comparison with real ones [10]. We also considered various transfer functions (TFs). Note that in contrast with Cirio et al., we focus on interactions with a virtual character and we wonder whether this joystick-based interface will induce natural adaptations of trajectories. We propose evaluating the 4 following TF, illustrated in Figure 6 left:

- $\mathbf{R}$ (Rotation) The longitudinal axis of the joystick controls speed $s$. Speed is bounded to $1.4 m \cdot s^{-1}$. The lateral axis of 

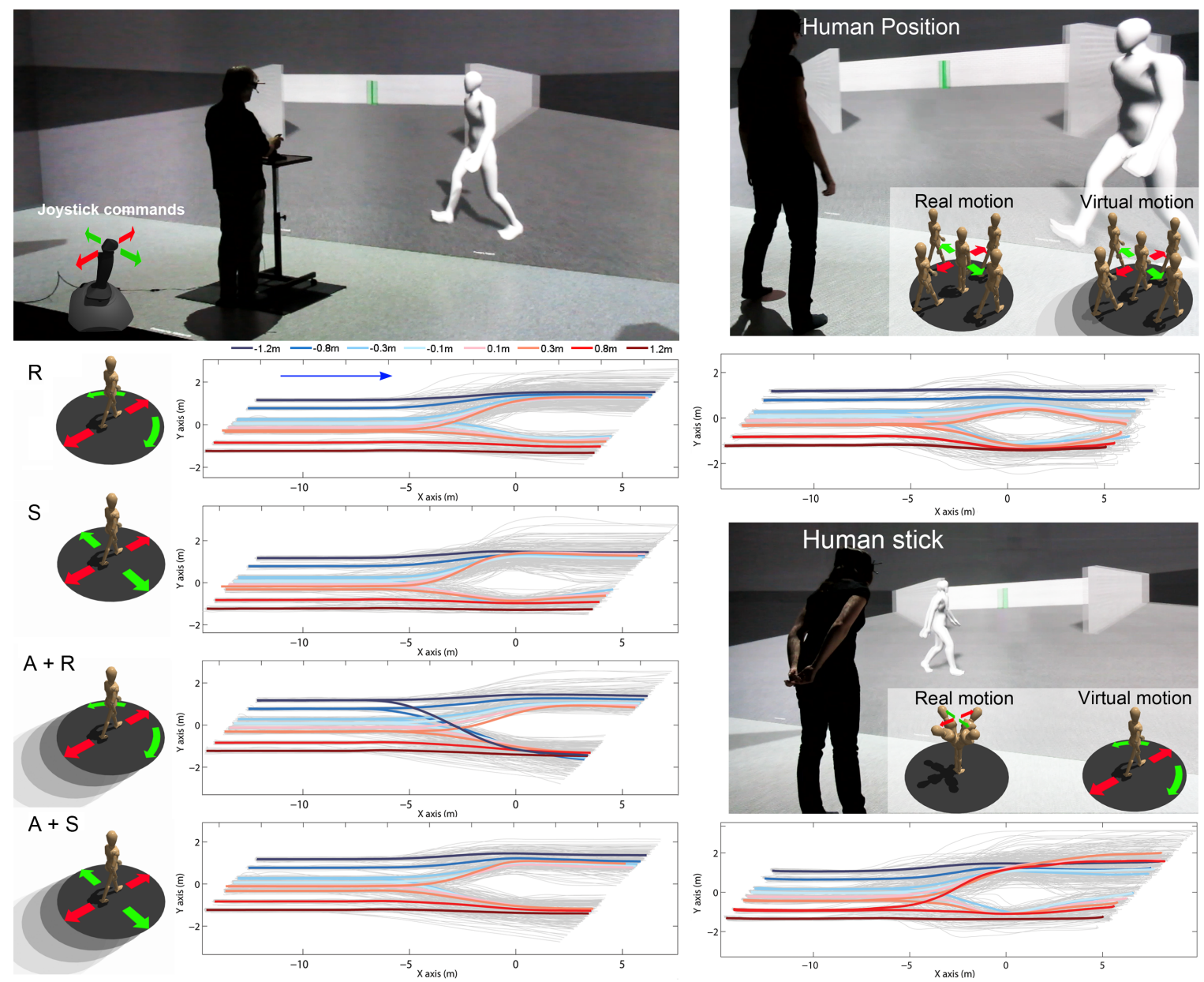

Fig. 6. Left: A participant interacting with a virtual character using a joystick-based interface. We considered 4 transfer functions which general principle and resulting locomotion traces relatively to the virtual character motion are illustrated. Right: A participant interacting with a virtual character using whole-body locomotion metaphors (HP and HS) and the associated traces of the resulting locomotion trajectories relative to the virtual character motion.

the joystick controls angular rotation speed $\dot{\theta}$ (bounded to $[-25,25]$ deg.s $\left.{ }^{-1}\right)$ :

$$
(s, \dot{\theta})_{\text {virt }}=(1.4,25) \cdot(x, y)_{j o y}
$$

where $x$ corresponds to the longitudinal axis of the joystick, and $y$ the lateral one $(x, y \in[-1,1])$.

- $\mathbf{S}$ (Side motion) in contrast with $\mathbf{R} \mathrm{TF}$, orientation is fixed, and the lateral axis controls the lateral speed $s_{l}$ of motion:

$$
\left(s, s_{l}\right)_{\text {virt }}=(1.4,1) \cdot(x, y)_{j o y}
$$

- $\mathbf{A}+\mathbf{R}$ (Automatic forward + Rotation) and A+S (Automatic forward + Side motion) TFs combine $\mathbf{R}$ and $\mathrm{S}$ TF with an automatic forward motion set at $1.4 m \cdot s^{-1}$. This means that users perform actions on the joystick only to make adaptations to their trajectories while they are automatically moved toward their goal.

$$
\begin{gathered}
\mathbf{A}+\mathbf{R}:(s, \dot{\theta})_{\text {virt }}=(0.6,25) \cdot(x, y)_{\text {joy }}+(1.4,0) \\
\mathbf{A}+\mathbf{S}:\left(s_{t}, s_{l}\right)_{\text {virt }}=(0.6,1) \cdot(x, y)_{\text {joy }}+(1.4,0)
\end{gathered}
$$

We have chosen these $4 \mathrm{TF}$ for the following reasons: $\mathrm{R}$ and $\mathrm{S}$ TFs are two different ways of controlling trajectory orientation. By adding the automatic motion $\mathbf{A +}$, we compare two different ways of controlling speed. Note that for A+ TFs, the participants act on the joystick only when they need to perform adaptations (when the joystick is released, motion is directed toward the goal, at comfortable speed). The joystick axis gains $\left(0.6,1 \mathrm{~m} . \mathrm{s}^{-1}, 25 \mathrm{deg} . \mathrm{s}^{-1}\right)$ for each TF were carefully chosen. In real avoidance, adaptations are bounded. The gains we chose are high enough to allow participants to reach those bounds, while they are as low as possible to allow participants to accurately control their motion and avoid jerky adaptations that provoke cybersickness.

Second, we also focus on other interfaces which require physical motions that involve the whole body. Indeed, joystick only requires hand movements that may not be representative of human dynamics. We evaluated two metaphors illustrated on Figure 6 right. The first one is the human position metaphor (HP) and is based on an automatic forward motion combined with offset translations:

$$
(x, y)_{\text {virtual }}=(1.4 t, 0)+(x, y)_{\text {real }}
$$

where $t$ is the time and $(x, y)_{\text {real }}$ the participant position (offset from rest position, marked on the floor by a brown circle as displayed in the companion video).

The second metaphor is named the human-stick metaphor 
(HS). Participants virtually move by leaning in the desired direction of motion.

$$
\left(s, s_{l}\right)=\left(k_{x}, k_{y}\right) \cdot(x, y)_{h e a d}
$$

where $s$ is the longitudinal virtual speed, $s_{l}$ the lateral one, and $(x, y)_{\text {head }}$ the position of the head (offset from rest position). $k_{x}$ and $k_{y}$ are gains: they are calibrated for each participant during training so that they feel comfortable with the technique. Note that during the training, no feedback was given to users.

\subsubsection{Procedure}

Each of the 6 locomotion interfaces (Joystick with Transfer Functions R, S, A+R, A+S and whole-body metaphors HS, HP) were each studied successively, but to avoid ordering effects, we used a latin square design to randomize the sequence of interfaces. Before experimenting each interface, participants could train as much time as they wanted in a dedicated environment (large room with traffic cones on the floor), then they trained on the avoidance task 6 times. For each locomotion interface participants performed 32 collision avoidance trials presented in a randomized order: 8 conditions of $m p d$, 2 sides and 2 repetitions. In total, 192 trials were performed $(\approx 1 \mathrm{~h} 15 \mathrm{~min})$. At the end of the experiment, participants filled a subjective questionnaire about each of the tested locomotion interfaces. The questionnaire measured the learning, usability, efficacy and satisfaction of interfaces through an adaptation of the USE questionnaire [25], which included the statements presented below. Users answered using a 7-point Likert scale from 1 (strongly disagree) to 7 (strongly agree).

\section{- Learning:}

- It was easy to learn to use this interface

- It was easy to remember how to use this interface

- Usability:

- It was easy to use this interface

- The motion resulting from this interface was intuitive

\section{- Efficacy:}

- This interface allows you to perform the task successfully

- The motion resulting from this interface was in accordance to the one you wanted to perform

\section{- Satisfaction:}

- You were satisfied to use this interface to perform the task

- This interface was pleasant to use

\subsubsection{Analysis}

For each trial, we recorded participants' trajectories. We computed the number of collisions with the virtual walker per participant. We computed for each trial the mpd as defined in Olivier et al. studies [31, 30]. We focused our analysis on the normalized interaction phase, between tsee $(0 \%)$, the first instant the user can see the virtual human, and tcross (100\%), the instant when the distance between the user and the virtual human is minimal. We studied $m p d$ only on trials for which the initial experimental conditions were respected ( $m p d$ (tsee) $\pm 20 \mathrm{~cm})$. We computed the derivative of $m p d$ on the normalized time and analysed its changes of sign to identify the main phases (no change, increase or decrease of the $m p d$ value) in mpd time evolution with respect to the locomotion interface.

Statistics were performed using Statistica Software. All effects were reported at $p<0.05$. Normality was assessed using a Kolomogorov Smirnov test. We performed analysis of variance (ANOVA) with repeated measures on the locomotion interface factors. The dependent variables were the number of collisions and minimum distance between the user and the virtual human, i.e., $m p d$ (tcross). Greenhouse-Geisser adjustments to the degrees of freedom were applied, when appropriate, to avoid any violation of the sphericity assumption. The effect size was computed using partial eta squared $\left(\eta_{p}^{2}\right)$. When appropriate, Bonferroni post-hoc tests were used to further analyse significant effects.

To compare initial and final values of $m p d$ during the interaction we used paired t-test for each initial experimental value of mpd (grouped by absolute value) and each locomotion interface. We also compared for each locomotion interface the general results of $m p d$ at tcross (all initial experimental conditions of $m p d$ gathered) with the ones obtained in [31] using independant t-test. We also compared for each locomotion interface the values of $m p d$ for several instants representing the change of sign of $m p d$ time derivative using a Wilcoxon signed rank test. Finally we analyzed the influence of the locomotion interface on the answers to the questionnaire using a Friedman test with a post hoc Wilcoxon signed rank test for each item.

\subsection{Results and Discussion}

Resulting trajectories relative to the virtual character motion are displayed in Figure 6 for each of the locomotion interfaces of the experiment. The number of collision avoidance failures, i.e. trials where there was a collision, is illustrated in Figure 7. There was an effect of the locomotion interface on the total number of collisions per participant $(F(2.1,29.3)=16.5, p<$ $0.0001, \eta_{p}^{2}=0.54$ ). Bonferroni post-hoc tests showed that there were more collisions with the HP interface compared to the other ones $(p<0.05)$, which makes it a bad candidate to match human behavior. This combination between real walking (to create offset position) and automatic virtual walking probably made the estimation of the self-position and the relative motion of the character too complex.

For each locomotion interface, average $m p d$ evolution during the interaction phase is displayed in Figure 8a. First, we analyzed if there were differences in $m p d$ values between the first instant of the interaction $0 \%$ (tsee) and the last instant $100 \%$ 


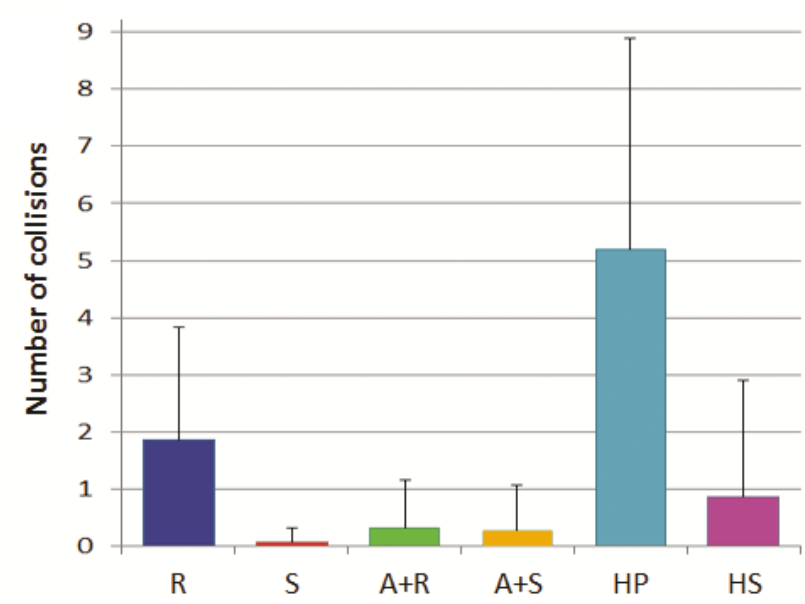

Fig. 7. Average number of collisions $( \pm S D$ ) with the virtual character per participant (among 32 trials per participant).

(tcross). Results of the paired t-tests are reported in Figure 8b. Results showed, for each initial experimental condition of $\mathrm{mpd}$ (gathered by absolute value of $m p d$ (tsee): $0.1,0.3,0.8$ and $1.2 \mathrm{~m}$ ) and for each locomotion interface, that $\operatorname{mpd}$ (tcross) was higher than $m p d$ (tsee). This means that users adapted their trajectories to avoid a collision with the virtual human in order to provide them with sufficient space at the crossing: the adaptations of the users' trajectories were then efficient to perform collision avoidance, which validates Hypothesis \#1.

Moreover, in real conditions, it was shown that walkers performed an adaptation of their trajectory to increase $m p d$ only when $m p d$ (tsee) was below $1 \mathrm{~m}$ [31]. In our experiments, we also observed an adaptation for a $1.2 \mathrm{~m}$ initial mpd value. Second, results showed that the crossing distance is influenced by the locomotion interface $(F(2.6,36.2)=$ $\left.6.21, p<0.005, \eta_{p}^{2}=0.3\right)$. Bonferroni post-hoc tests showed that $m p d$ (tcross) value is lower for HP interface compared to the other ones $(p<0.05)$. Finally, multiple t-test comparisons between the crossing distance reported in real conditions $(\operatorname{mpd}($ tcross $)=0.84 \pm 0.19 \mathrm{~m})[31]$ and the crossing distance obtained for each virtual locomotion interface showed a significant difference where mpd(tcross) is higher for all of the virtual conditions in comparison with the real ones $(p<0.001)$. In conclusion, we show that, in comparison with reality, users adapt their locomotion for lower risk of future collision (absence of adaptation would lead to a collision free motion), and that they perform larger adaptations which result into larger passage distance in VR. We interpret that this difference in the adaptation threshold could be a consequence of the compression of distance perception in VR.

For each locomotion interface, average time derivative of mpd during the interaction phase is displayed in Figure 9a. For each curve, we identified all the instants corresponding to a change of sign of $m p d$ time derivative and we computed the corresponding mpd values. Then, we applied Wilcoxon signed rank tests to compare the $m p d$ values which encompass these changes of sign to characterize the evolution of $m p d$ (no change, increase, decrease). Results of these tests are reported on Figure 9b. Results from the collision avoidance between

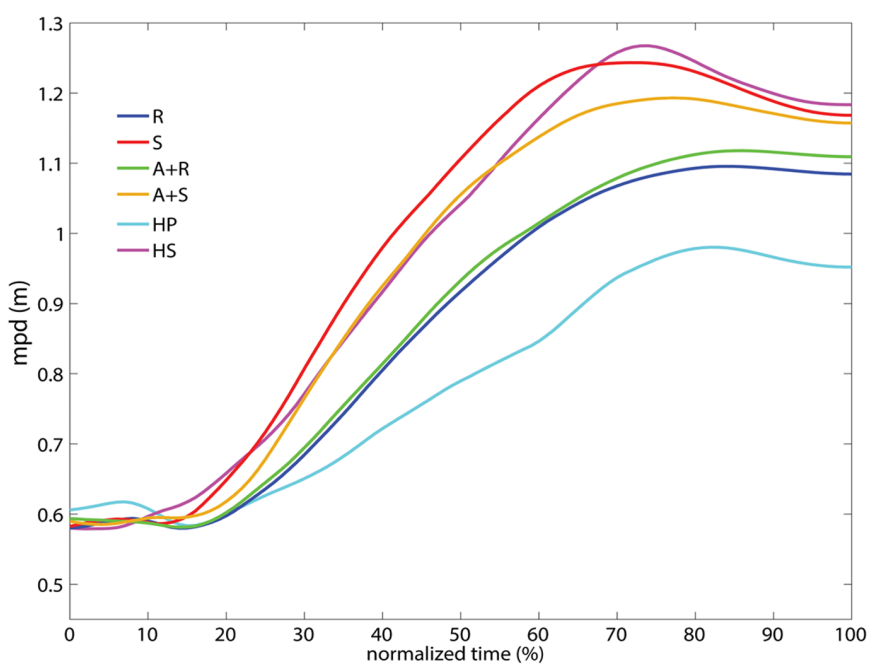

\begin{tabular}{|c|c|c|c|c|c|c|}
\hline & \multicolumn{3}{|c|}{$0.1 \mathrm{~m}$} & \multicolumn{3}{|c|}{$0.3 \mathrm{~m}$} \\
\hline & $\mathrm{mpd}(\mathrm{tsee})$ & $\mathrm{mpd}(\mathrm{tcross})$ & Paired $\mathrm{t}$-test & $\mathrm{mpd}(\mathrm{tsee})$ & $\mathrm{mpd}$ (tcross) & Paired t-test \\
\hline $\mathrm{R}$ & $0.11 \pm 0.07$ & $0.88 \pm 0.38$ & $t=20.06^{*}$ & $0.30 \pm 0.08$ & $0.94 \pm 0.38$ & $t=15.76^{*}$ \\
\hline $\mathrm{s}$ & $0.12 \pm 0.07$ & $1.05 \pm 0.42$ & $t=20.87^{*}$ & $0.29 \pm 0.09$ & $1.09 \pm 0.41$ & $\mathrm{t}=18.34^{*}$ \\
\hline$A+R$ & $0.12 \pm 0.07$ & $0.90 \pm 0.25$ & $t=30.86^{*}$ & $0.30 \pm 0.08$ & $0.97 \pm 0.27$ & $\mathrm{t}=25.56^{*}$ \\
\hline$A+S$ & $0.11 \pm 0.06$ & $0.99 \pm 0.26$ & $t=33.24^{*}$ & $0.29 \pm 0.07$ & $1.03 \pm 0.0,26$ & $t=26.75^{*}$ \\
\hline HP & $0.12 \pm 0.07$ & $0.71 \pm 0.72$ & $\mathrm{t}=8.35^{*}$ & $0.31 \pm 0.08$ & $0.74 \pm 0.39$ & $\mathrm{t}=10.84^{*}$ \\
\hline \multirow[t]{3}{*}{ HS } & $0.14 \pm 0.09$ & $1.07 \pm 0.40$ & $t=20.86^{*}$ & $0.29 \pm 0.13$ & $1.07 \pm 0.33$ & $t=19.12^{*}$ \\
\hline & \multicolumn{3}{|c|}{$0.8 \mathrm{~m}$} & \multicolumn{3}{|c|}{$1.2 \mathrm{~m}$} \\
\hline & $\mathrm{mpd}($ tsee $)$ & $\mathrm{mpd}$ (tcross) & Paired t-test & $\mathrm{mpd}(\mathrm{tsee})$ & $\mathrm{mpd}$ (tcross) & Paired t-tes \\
\hline $\mathrm{R}$ & $0.80 \pm 0.07$ & $1.01 \pm 0.15$ & $\mathrm{t}=8.96^{*}$ & $1.19 \pm 0.09$ & $1.39 \pm 0.24$ & $\mathrm{t}=7.87^{*}$ \\
\hline $\mathrm{s}$ & $0.78 \pm 0.07$ & $1.16 \pm 0.31$ & $\mathrm{t}=10.50^{*}$ & $1.20 \pm 0.07$ & $1.38 \pm 0.22$ & $\mathrm{t}=6.97^{*}$ \\
\hline$A+R$ & $0.81 \pm 0.07$ & $1.19 \pm 0.31$ & $\mathrm{t}=11.54^{*}$ & $1.21 \pm 0.08$ & $1.40 \pm 0.27$ & $\mathrm{t}=6.91^{*}$ \\
\hline$A+S$ & $0.80 \pm 0.07$ & $1.21 \pm 0.30$ & $\mathrm{t}=12.98^{*}$ & $1.20 \pm 0.07$ & $1.41 \pm 0.26$ & $t=7.46^{*}$ \\
\hline HP & $0.81 \pm 0.08$ & $1.12 \pm 0.33$ & $\mathrm{t}=8.85^{*}$ & $1.21 \pm 0.07$ & $1.33 \pm 0.24$ & $\mathrm{t}=4.68^{*}$ \\
\hline HS & $0.76 \pm 0.12$ & $1.19 \pm 0.34$ & $\mathrm{t}=9.48^{*}$ & $1.21 \pm 0.13$ & $1.43 \pm 0.33$ & $\mathrm{t}=5.48^{*}$ \\
\hline
\end{tabular}

Fig. 8. a) Average mpd evolution in time during interaction. b) Results of the paired t-test comparisons between $m p d$ (tsee) and $m p d$ (tcross) ( ${ }^{\star * *}$ means $p<0.001)$.

two real walkers [31] are reported on the top of Figure $9 \mathrm{~b}$ for comparison purpose. The first main result is that for all locomotion interfaces, we can observe that collision avoidance task is solved by anticipation. Indeed, mpd increase (in red on the figure) is over before the crossing. As in real conditions, $m p d$ is even slightly decreased (in blue) but maintained to a comfortable value. This validates Hypothesis \#2. When considering the whole duration of interaction, results showed that collision avoidance was strictly temporally structured as in real conditions (observation (green), reaction (red), regulation (blue)) only for the Joystick with A+R TF and the HS interface.

The analysis of questionnaires, illustrated in Figure 10, did not show any effect of the locomotion interface on the efficacy item $\left(\chi^{2}(5)=9.68, p=0.085\right)$, even if the HP interface led to higher collisions with the virtual human. However, the locomotion interface significantly influenced the users' answers on the learning $\left(\chi^{2}(5)=15,97, p=0.0069\right)$, usability $\left(\chi^{2}(5)=21.65, p=0.00061\right)$ and satisfaction $\left(\chi^{2}(5)=\right.$ $16.12, p=0.0065)$ items. Post hoc analyses showed that participants preferred the A+S TF compared to HP and the HS ones $(p<0.01)$ for learning. Users found that HS interface was 


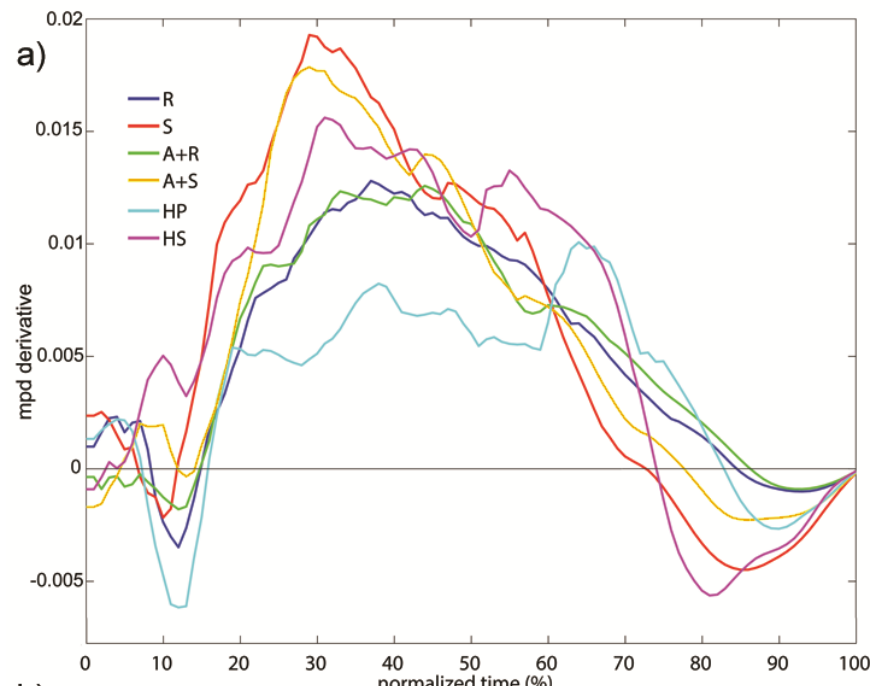

b)

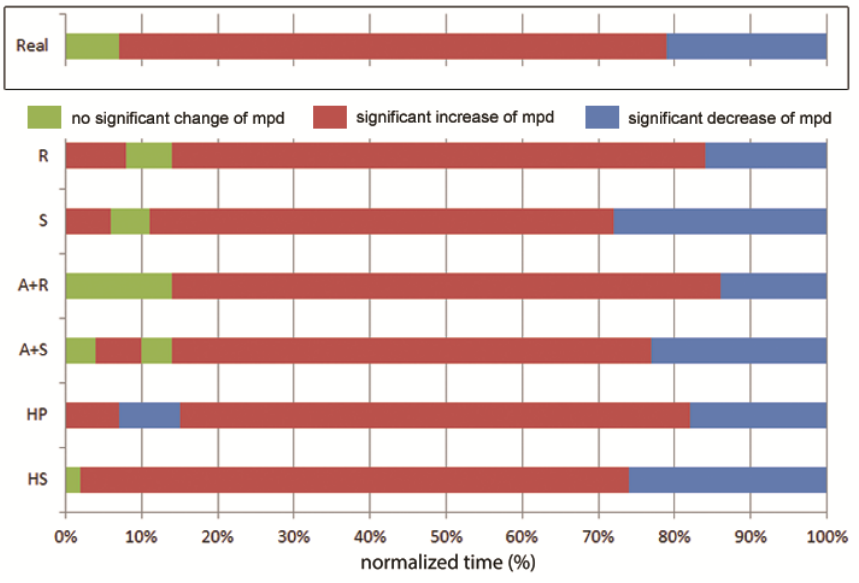

Fig. 9. a) Average time derivative of $m p d$ for each locomotion interface. b) Results of the Wilcoxon signed-rank test comparisons concerning the evolution of $m p d$ between each change of sign of its time derivative. Results for real conditions are reported from [31]

lower in term of usability than the 4 joystick TFs $(p<0.05)$. Finally, users were less satisfied with the HS interface than with the joystick associated with $A+R$ and $A+S$ TFs and with the HP interface $(p<0.05)$. From this analysis, it appears the HS locomotion interface was less appreciated than the other ones. We believe that the main reason is that it requires more physical effort than the joystick and the HP interface(freely reported by 5 participants: "the HS was tiring”).

\section{General Discussion}

Can Virtual Reality be used to study the kinematics of locomotion interactions, such as collision avoidance? We should remind that VR offers a unique opportunity to deeply understand local interactions between humans during locomotion. Such interactions are difficult to study in real conditions, because interactions depend on relative kinematics parameters (relative distance, motion, speed or angle), which cannot be easily controlled and repeated over trials. Nevertheless, understanding how humans respond in those situations of interactions is crucial to design crowd simulators: microscopic approaches are based on models of local interactions between people. The

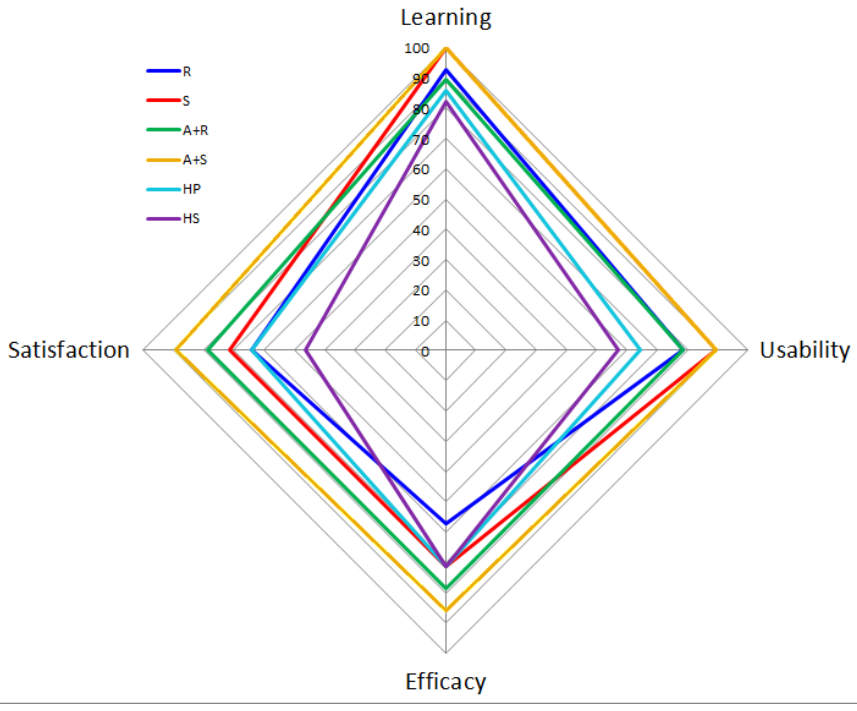

Fig. 10. Subjective feedback of participants with respect to the locomotion interfaces in term of learning, usability, efficacy and satisfaction aspects. Results are presented as median values of the users' answers.

crowd simulation application field is large, ranging from architecture design to the entertainment industry. Because we are able to accurately control a virtual character motion, and because we can also control it with respect to the participant motion, VR facilitates the full control of some complex situations of interaction between humans, with the ability to accurately repeat them over several subjects. An example of such a study is presented in [7]. There is no easy way to control the relative motions of subjects in real experimental conditions. The question is the validity of experimental data acquired using VR.

We proposed two experiments to address the question of usability of VR to perform acquisition of kinematics data on interactions, in which we considered the trajectory formed by participants immersed in situations of local interactions with a virtual character. We studied the locomotion trajectories formed by the participants using various locomotion interfaces. We compared some characteristics of these trajectories with real ones.

Our study is decomposed in 2 steps. In a first step, we evaluate the user's perception of the collision risk with a moving virtual character. We showed that the risk of collision is mostly perceived with a high level accuracy when participants have enough time to evaluate the situation. There are two exceptions, when $m p d$ equals $-0.8 m$ and $0.3 m$. These values corresponds to situation where the future distance of approach is close to distance of contact: this is not surprising to find some false answers around this value. However, wrong answers are not symmetric with the passage order. When the character pass behind $(m p d=-0.8 m)$, participants provide false positive answer (erroneous estimation of a risk of collision). At the opposite, when the character pass in front $(m p d=0.3 m)$, participants provide false negative answers (erroneous estimation of absence of risk of collision). Our interpretation is that participants estimate their own position in the virtual space with a forward shift: they estimate to be about tens centimeter ahead their actual position.

In a second step, we compare collision avoidance metrics 
with the ones observed in real conditions by considering a joystick with various control laws or body-based locomotion interfaces. All the studied locomotion interfaces led to qualitatively realistic trajectories, with some quantitative differences in comparison with real avoidance behaviors. In particular, participants estimated their position with roughly ten centimeters shift, they slightly over-adapted their trajectories (i.e., they increased clearance). Those differences may result from mistakes in distance perception in VR $[21,23,35]$.

Our results show that VR is a relevant tool to acquire kinematics data. Indeed, our most important conclusion is that, in the situation we studied, users had a similar behaviors between real conditions or virtual ones. This means that they performed the same successive phases during avoidance (observation, anticipated avoidance maneuvers, regulation of the crossing distance), they respected the initial order of passage at the crossing point as expected, etc. These results which highlight strong similarities between avoidance behaviors in real and virtual conditions can be linked to the results of Cirio et al. [10] who observed that users control the same way their trajectory in virtual environments than in real environments to achieve goal-oriented locomotion tasks. We thus recommend corroborating VR measurements with real empirical data if quantitative analysis is required. A quantitative analysis to compare real and virtual conditions of interactions has been presented in [1]: this study also shows that users adapt their trajectory differently when avoiding a virtual object or a virtual character, just as they do in real conditions.

More precisely, we showed that the joystick device combined with $\mathrm{A}+\mathrm{R}$ TF matches best with human behavior observed in real conditions, which makes it a good candidate for natural walking and interaction with other people. Some limitations of the tested interfaces can nonetheless be identified. The interfaces that include an automatic motion component $(\mathrm{A}+\mathrm{R}, \mathrm{A}+\mathrm{S}$, HP) constrained adaptations around the "ideal speed" which is automatically followed. Note that R and A+R TFs are the only ones that enable participants to freely navigate in the entire environment (orientation is constant with the two other techniques). $\mathrm{R}$ and $\mathrm{S}$ TFs for joystick-based techniques are also limited, because speed adaptations by accelerating could not be performed, users pushed the joystick fully ahead from the beginning. HS metaphor does not have such limitations, but users disliked it, and it resulted in the most important quantitative differences with real situations.

As a second conclusion, we are able to provide VR platform designers with guidelines to choose the most adequate locomotion interface. Generally speaking, this article provides a full method to evaluate how realistically users can behave in interactions during locomotion tasks. Among the interfaces we tested, we can recommend some depending on the type of locomotion task experimenters want to study. For simple locomotion task (e.g., go ahead) with limited adaptations, joystick with $\mathrm{A}+\mathrm{R} \mathrm{TF}$ is the best option. For more complex tasks and adaptations, joystick with $\mathrm{R} T \mathrm{TF}$ is recommended. As a second conclusion, we are able to provide VR platform designers with guidelines to choose the most adequate locomotion interface. Generally speaking, this article provides a full method to evaluate how realistically users can behave in interactions dur- ing locomotion tasks. Among the interfaces we tested, we can recommend some depending on the type of locomotion task experimenters want to study. For simple locomotion task (e.g., go ahead) with limited adaptations, joystick with $\mathrm{A}+\mathrm{R} \mathrm{TF}$ is the best option. For more complex tasks and adaptations, joystick with $\mathrm{R} T F$ is recommended.

\section{Conclusion}

In this article, we study and discuss the use of Virtual Reality to observe humans during locomotion and interaction tasks. Our results show that Virtual Reality offers opportunities for qualitative analysis of such human behaviors and opens several perspectives. First, we had to restrict the number of studied interfaces for experimental reasons. We considered the most commonly used ones, especially the joystick. This is few in comparison with the numerous existing ones (for example, omnidirectional treadmills would be relevant). We also focused on a few transfer functions associated with the joystick device and additional work should be relevant for a better understanding of what aspect of the function relates to how humans move.

Second, we did not consider head-mounted displays which are interesting since they allow participants to walk in a large environment. However, they suffer from limited peripheral vision (used to detect future risks of collision) or low resolution displays. Moreover they need very accurate tracking of orientations, and can provoke cybersickness. The use of a CAVE was then suitable for this first validation of the platform, and future investigation is required for HMD interfaces.

Third, we studied interactions with a passive character. The reason was that we wanted to expose all the users to exactly the same situations. This is a key aspect to standardize the experimental conditions. Moreover, if a reactive character is wanted, the question of the algorithm to steer the character's motion and of its level of realism is a difficult one. We could have used a moving cylinder to make it more obvious that only the user would perform the collision avoidance task. However, users were informed about the passiveness of the virtual walker and they should not expect cooperation which should reduce the bias induced by such a virtual obstacle. Moreover, participants started adapting their trajectories once the collision risk was detected, before any adaptation from the virtual character can be expected. Thus only the final part of the interaction would be impacted. Indeed, the quantity of adaptations is undoubtedly higher with a passive character because only the participants perform trajectory adaptations.

There is still a lot of investigation to perform, not only about the whole trajectory, but also about the clues conveyed by head and shoulder orientations, eye contact, etc. It is probably important and relevant to animate characters so that they accurately display those clues, probably more than questions about character responsiveness and ability to adapt his locomotion trajectory.

More generally, human behaviors in crowds are poorly understood now. Crowd behaviors studies in real conditions are extremely complex to perform. We believe that VR provides a unique opportunity to study individual behaviors in crowds and could provide a deep understanding of the process by which human control their motion in such a context. Following this 
research path, we may expect large progress for microscopic crowd simulation algorithm in terms of realism, as well as extension of their application field.

\section{ACKNOWLEDGMENTS}

The authors wish to thank the Immersia Team and Benjamin Ray for their technical help as well as all the volunteers for the experiment. This work is funded by the French National Research Agency ANR, project PERCOLATION number ANR13-JS02-0008.

\section{REFERENCES}

[1] F. Argelaguet Sanz, A.-H. Olivier, G. Bruder, J. Pettre, and A. Lecuyer. Virtual proxemics: Locomotion in the presence of obstacles in large immersive projection environments. In IEEE Virtual Reality, Arles, France, Mar. 2015.

[2] T. Banton, J. Stefanucci, F. Durgin, A. Fass, and D. Proffitt. The perception of walking speed in a virtual environment. Presence, 14(4):394-406, Aug 2005.

[3] P. Basili, M. Sağlam, T. Kruse, M. Huber, A. Kirsch, and S. Glasauer. Strategies of locomotor collision avoidance. Gait \& posture, 37(3):385-390, 2013.

[4] B. Bideau, R. Kulpa, N. Vignais, S. Brault, F. Multon, and C. Craig. Using virtual reality to analyze sports performance. Computer Graphics and Applications, IEEE, 30(2):14-21, 2010.

[5] R. W. Bohannon. Comfortable and maximum walking speed of adults aged 2079 years: reference values and determinants. Age and ageing, 26(1):15-19, 1997.

[6] G. Bruder, P. Wieland, B. Bolte, M. Lappe, and F. Steinicke. Going with the flow: Modifying self-motion perception with computer-mediated optic flow. In Mixed and Augmented Reality (ISMAR), 2013 IEEE International Symposium on, pages 67-74. IEEE, 2013.

[7] J. Bruneau, A.-H. Olivier, and J. Pettré. Going through, going around: A study on individual avoidance of groups. Visualization and Computer Graphics, IEEE Transactions on, 21(4):520-528, 2015

[8] B. J. Chihak, J. M. Plumert, C. J. Ziemer, S. Babu, T. Grechkin, J. F. Cremer, and J. K. Kearney. Synchronizing self and object movement: how child and adult cyclists intercept moving gaps in a virtual environment. Journal of experimental psychology: human perception and performance, 36(6):1535, 2010.

[9] M. E. Cinelli and A. E. Patla. Locomotor avoidance behaviours during a visually guided task involving an approaching object. Gait \& posture, 28(4):596-601, 2008.

[10] G. Cirio, A.-H. Olivier, M. Marchal, and J. Pettré. Kinematic evaluation of virtual walking trajectories. IEEE Transactions on Visualization and Computer Graphics, 19(4):671-680, Apr. 2013.

[11] J. E. Cutting, P. M. Vishton, and P. A. Braren. How we avoid collisions with stationary and moving objects. Psychological review, 102(4):627-651, 1995.

[12] T. Ducourant, S. Vieilledent, Y. Kerlirzin, and A. Berthoz. Timing and distance characteristics of interpersonal coordination during locomotion. Neuroscience letters, 389(1):6-11, 2005.

[13] B. R. Fajen and W. H. Warren. Behavioral dynamics of steering, obstable avoidance, and route selection. Journal of Experimental Psychology: Human Perception and Performance, 29(2):343-362, 2003

[14] P. W. Fink, P. S. Foo, and W. H. Warren. Obstacle avoidance during walking in real and virtual environments. ACM Trans. Appl. Percept., 4(1), Jan. 2007.

[15] M. Gérin-Lajoie, C. Richards, and B. McFadyen. The negociation of stationary and moving obstructions during walking: anticipatorylocomotor adaptations and preservation of personal space. Motor Control, 9:242-269, 2005

[16] M. Gérin-Lajoie, C. L. Richards, J. Fung, and B. J. McFadyen. Characteristics of personal space during obstacle circumvention in physical and virtual environments. Gait \& Posture, 27(2):239-247, 2008

[17] J. Hollerbach. Locomotion interfaces, chapter Handbook of Virtual Environments: Design, Implementation, and Applications, pages 239-254. Lawrence Erlbaum Associates, 2002.

[18] J. H. Hollman, R. H. Brey, T. J. Bang, and K. R. Kaufman. Does walking in a virtual environment induce unstable gait?: An examination of vertical ground reaction forces. Gait \& Posture, 26(2):289-294, 2007.

[19] M. Huber, Y.-H. Su, M. Krüger, K. Faschian, S. Glasauer, and J. Hermsdörfer. Adjustments of speed and path when avoiding collisions with another pedestrian. PloS one, 9(2):e89589, 2014.

[20] I. Karamouzas and M. Overmars. Simulating the local behaviour of small pedestrian groups. In Proceedings of the 17th ACM Symposium on Virtual Reality Software and Technology, VRST '10, pages 183-190, New York, NY, USA, 2010. ACM.

[21] D. R. Lampton, D. P. McDonald, M. Singer, and J. P. Bliss. Distance estimation in virtual environments. Proceedings of the Human Factors and Ergonomics Society Annual Meeting, 39(20):1268-1272, 1995.

[22] S. Lemercier, A. Jelic, R. Kulpa, J. Hua, J. Fehrenbach, P. Degond, C. AppertRolland, S. Donikian, and J. Pettré. Realistic following behaviors for crowd simulation. Comp. Graph. Forum, 31(2pt2):489-498, May 2012.

[23] J. Loomis and J. Knapp. Visual perception of egocentric distance in real and virtual environments. Virtual and adaptive environments: Applications, implications, and human performance issues, pages 21-46, 2003.

[24] J. M. Loomis, J. J. Blascovich, and A. C. Beall. Immersive virtual environment technology as a basic research tool in psychology. Behavior Research Methods, Instruments, \& Computers, 31(4):557-564, 1999.
[25] A. M. Lund. Measuring usability with the use questionnaire. Usability interface, $8(2): 3-6,2001$

[26] H. A. Mallot, S. Gillner, H. A. van Veen, and H. H. Bülthoff. Behavioral experiments in spatial cognition using virtual reality. In Spatial cognition, pages 447-467. Springer, 1998.

[27] B. J. Mohler, S. H. Creem-Regehr, and W. B. Thompson. The influence of feedback on egocentric distance judgments in real and virtual environments. In Proceedings of the 3 rd symposium on Applied perception in graphics and visualization, pages 9-14. ACM, 2006.

[28] B. J. Mohler, S. H. Creem-Regehr, W. B. Thompson, and H. H. Bulthoff. The effect of viewing a self-avatar on distance judgments in an HMD-Based virtual environment. Presence: Teleoperators and Virtual Environments, 19:230-242, 2010.

[29] C. Neth, J. Souman, D. Engel, U. Kloos, H. Bülthoff, and B. Mohler. Velocitydependent curvature gain and avatar use for redirected walking. In 2010 Joint Virtual Reality Conference of EuroVR-EGVE-VEC (JVRC 2010), 2010.

[30] A.-H. Olivier, A. Marin, A. Crétual, A. Berthoz, and J. Pettré. Collision avoidance between two walkers: Role-dependent strategies. Gait \& Posture, 38(4):751 - 756, 2013

[31] A.-H. Olivier, A. Marin, A. Crétual, and J. Pettré. Minimal predicted distance: A common metric for collision avoidance during pairwise interactions between walkers. Gait \& Posture, 36(3):399 - 404, 2012.

[32] A. E. Patla, S. Rietdyk, C. Martin, and S. Prentice. Locomotor patterns of the leading and the trailing limbs as solid and fragile obstacles are stepped over: some insights into the role of vision during locomotion. Journal of motor behavior, 28(1):35-47, 1996.

[33] J. Perrinet, A.-H. Olivier, and J. Pettré. Walk with me: Interactions in emotional walking situations, a pilot study. In Proceedings of the ACM Symposium on Applied Perception, SAP'13, pages 59-66, New York, NY, USA, 2013. ACM.

[34] J. Pettré, O. Siret, M. Marchal, J.-B. de la Rivire, and A. Lécuyer. Joyman: An immersive and entertaining interface for virtual locomotion. In SIGGRAPH Asia 2011 Emerging Technologies, SA '11, pages 22:1-22:1, New York, NY, USA, 2011. ACM.

[35] J. M. Plumert, J. K. Kearney, J. F. Cremer, and K. Recker. Distance perception in real and virtual environments. ACM Transactions on Applied Perception (TAP), 2(3):216-233, 2005

[36] S. Razzaque. Redirected Walking. PhD thesis, Chapel Hill, NC, USA, 2005. AAI3190299.

[37] R. S. Renner, B. M. Velichkovsky, and J. R. Helmert. The perception of egocentric distances in virtual environments - a review. ACM Comput. Surv., 46(2):23:1-23:40, Dec. 2013.

[38] R. A. Ruddle, E. Volkova, and H. H. Bülthoff. Learning to walk in virtual reality. ACM Trans. Appl. Percept., 10(2):11:1-11:17, June 2013.

[39] M. Slater, M. Usoh, and A. Steed. Taking steps: the influence of a walking technique on presence in virtual reality. ACM Trans. Comput.-Hum. Interact., 2(3):201-219, 1995

[40] J. L. Souman, P. R. Giordano, M. Schwaiger, I. Frissen, T. Thümmel, H. Ulbrich, A. D. Luca, H. H. Bülthoff, and M. O. Ernst. Cyberwalk: Enabling unconstrained omnidirectional walking through virtual environments. ACM Trans. Appl. Percept., 8(4):25:1-25:22, Dec. 2008.

[41] F. Steinicke, G. Bruder, T. Ropinski, and K. Hinrichs. Moving towards generally applicable redirected walking. In Proceedings of the Virtual Reality International Conference (VRIC), pages 15-24. Citeseer, 2008.

[42] F. Steinicke, Y. Visell, J. Campos, and A. Lécuyer. Human walking in virtual environments. Springer, 2013.

[43] E. Suma, S. Finkelstein, M. Reid, S. Babu, A. Ulinski, and L. Hodges. Evaluation of the cognitive effects of travel technique in complex real and virtual environments. IEEE Transactions on Visualization and Computer Graphics, 16(4):690-702, 2010.

[44] L. Terziman, M. Marchal, M. Emily, F. Multon, B. Arnaldi, and A. Lécuyer. Shakeyour-head: Revisiting walking-in-place for desktop virtual reality. In Proceedings of the 17th ACM Symposium on Virtual Reality Software and Technology, VRST '10, pages 27-34, New York, NY, USA, 2010. ACM.

[45] L. A. Vallis and B. J. McFadyen. Locomotor adjustments for circumvention of an obstacle in the travel path. Experimental brain research, 152(3):409-414, 2003.

[46] B. J. van Basten, S. E. Jansen, and I. Karamouzas. Exploiting motion capture to enhance avoidance behaviour in games. In Motion in Games, pages 29-40. Springer, 2009.

[47] W. H. Warren, S. Di, and B. R. Fajen. Behavioral dynamics of avoiding a moving obstacle. Journal of Vision, 3(9):134-134, 2003.

[48] W. H. Warren and B. R. Fajen. Optic Flow and Beyond, chapter From optic flow to laws of control, pages 307-337. Kluwer (Editors: L. M. Vaina, S. A. Beardsley, and S. Rushton), 2004

[49] M. Whitton, J. Cohn, J. Feasel, P. Zimmons, S. Razzaque, S. Poulton, B. McLeod, and J. Brooks, F.P. Comparing VE locomotion interfaces. In IEEE Virtual Reality, pages 123-130, 2005.

[50] P. Willemsen, M. B. Colton, S. H. Creem-Regehr, and W. B. Thompson. The effects of head-mounted display mechanics on distance judgments in virtual environments. In Proceedings of the 1st Symposium on Applied Perception in Graphics and Visualization, APGV '04, pages 35-38, New York, NY, USA, 2004. ACM.

[51] C. Zanbaka, B. Lok, S. Babu, A. Ulinski, and L. Hodges. Comparison of path visualizations and cognitive measures relative to travel technique in a virtual environment. IEEE Transactions on Visualization and Computer Graphics, 11(6):694 -705, 2005. 